\title{
Long-Term Effects Secondary to Cancer Therapy
}

National Cancer Institute

\section{Source}

National Cancer Institute. Long-Term Effects Secondary to Cancer Therapy. NCI

Thesaurus. Code C115226.

Side effects of cancer treatment occurring a long time after the treatment was completed. 